\title{
‘Exotic vector-like pair’ of color-triplet scalars
}

\author{
Andrea Addazi \\ Dipartimento di Fisica, Università di L'Aquila, \\ 67010 Coppito $A Q$, Italy \\ LNGS, Laboratori Nazionali del Gran Sasso, \\ 67010 Assergi AQ, Italy \\ E-mail: andrea.addazi@infn.lngs.it
}

ABstract: We propose a minimal extension of Standard Model, generating a Majorana mass for neutron, connected with a mechanism of Post-Sphaleron Baryogenesis. We consider an 'exotic vector-like pair' of color-triplet scalars, an extra Majorana fermion $\psi$, and a scalar field $\phi$, giving mass to $\psi$. The vector-like pair is defined 'exotic' because of a peculiar mass term of the color-triplet scalars, violating Baryon number as $\Delta B=1$. Such a mass term could be generated by exotic instantons in a class of string-inspired completions of the Standard Model: open (un-)oriented strings attached between D-brane stacks and Euclidean D-branes. A Post-Sphaleron Baryogenesis is realized through $\phi$-decays into six quarks (antiquarks), or through $\psi$-decays into three quarks (antiquarks). This model suggests some intriguing B-violating signatures, testable in the next future, in NeutronAntineutron physics and LHC. We also discuss limits from FCNC. Sterile fermion can also be light as $1-100 \mathrm{GeV}$. In this case, the sterile fermion could be (meta)-stable and $n-\bar{n}$ oscillation can be indirectly generated by two $n-\psi, \psi-\bar{n}$ oscillations, without needing of an effective Majorana mass for neutron. Majorana fermion $\psi$ can be a good candidate for WIMP-like dark matter.

KeYwords: Supersymmetry Phenomenology, Strings and branes phenomenology

ArXiv EPRINT: 1501.04660 


\section{Contents}

1 Introduction 1

2 A model for a neutron Majorana mass 2

2.1 FCNC bounds and the space of the parameters 4

$\begin{array}{lll}3 & \text { LHC physics } & 6\end{array}$

4 Post-sphaleron baryogenesis $\quad 6$

$\begin{array}{lll}4.1 & \text { Scalar-decays into six quarks (antiquarks) } & 7\end{array}$

4.2 Majorana fermion decays in three quarks (antiquarks) 9

5 Beyond the toy-model: string-inspired standard model and exotic instan$\begin{array}{ll}\text { tons } & 10\end{array}$

6 Conclusions 12

\section{Introduction}

Has the neutron a Majorana mass or not? This is not just an academic question. Majorana himself proposed in '37', that neutron could have a Majorana mass term $\delta m n n+h . c[1]$. We do not know if Majorana understood immediately the depth of his proposal; but today we get that existence of a "Majorana's fermion" is related to baryon or lepton numbers' violations. In particular, a Majorana mass for neutron implies a neutron-antineutron transition, violating baryon number by $\Delta B=2[2-4]$. The current limit on $n-\bar{n}$ is $\tau_{n \bar{n}}=1 / \delta m>0.86 \times 10^{8}$ s with $90 \%$ C.L., implying $\delta m<7.7 \times 10^{-24} \mathrm{eV}$ [5]. This corresponds to a constraint $\mathcal{M}>300 \mathrm{TeV}$ on the effective operator $(u d d)^{2} / \mathcal{M}^{5}$. This limit is particularly loose with respect to other rare processes violating Baryon or Lepton numbers: $\tau_{n \bar{n}}>3 y r$ for neutron-antineutron can be compared with $\tau_{p \text {-decay }} \sim 10^{34 \div 35}$ yr for the Proton decays, $\tau_{0 \nu \beta \beta}>10^{25} \mathrm{yr}$ for neutrinoless double beta decays [6]. For these reasons, neutron-antineutron is becoming more and more an interesting challenge for model building $[20-26],{ }^{1}$ also considering possibility in the next future to enhance best limit of a factor 100: $\tau_{n \bar{n}}>10^{10} \mathrm{~s}$, testing $1000 \mathrm{TeV}$ scale [7]. (For a recent review about phenomenology of Baryon and Lepton violations, see also [27]). In this paper, we would like to suggest a simple minimal model connecting the "Majorana's question" with a mechanism of Baryogenesis. Depending on the particular region of the parameters, this model connects neutronantineutron physics with LHC, predicting a new peculiar phenomenology in collider physics. This model does not produce proton decay, and FCNC can be sufficiently suppressed.

\footnotetext{
${ }^{1}$ See also [42] for a short discussion about Neutron-Antineutron physics as a test of a new fifth force interaction (a more complete version is in preparation [43]).
} 
The main model's feature: we introduce an 'exotic' mixing mass term for a vectorlike pair of color scalar triplets, violating baryon number as $\Delta B=1$, i.e one color-triplet scalar has a different baryon number with respect to the other triplet antiscalar by exactly one unit. One scalar triplet has $B=1 / 3$, and the other has $B=2 / 3$. We call this an 'exotic vector-like pair'. We propose that existence of a $\Delta B=2$ Majorana Mass could be connected to a $\Delta B=1$ exotic mass term! In a broad sense, we have a seesaw mechanism for neutron, involving a non-diagonal mass matrix for scalars rather than fermions. ${ }^{2,3}$ This model is inspired by proposals in [28, 29, 53-56]: (NMS-)SM is obtained as a low energy limit of open (un)-oriented strings, attached between D-brane stacks and Euclidean D-branes. Euclidean D-branes are exotic stringy instantons, that can induce new non-perturbative mass terms, violating vector-like $\mathrm{U}(1) \mathrm{s}$, rather than axial-ones. In particular, in $[28,29], R$-parity is dynamically broken by exotic instantons, producing only particular B-violating operators, such as a mass term for a vector-like pair Proton decay is automatically suppressed in this model [28, 29].

An exotic vector-like pairs could be not only indirectly searched in $n-\bar{n}$ physics, but also at LHC, with peculiar processes: $p p \rightarrow j j \mathbb{E}_{T}$, for example, could be a spectacular signature of exotic vector-like pairs and dark matter.

This model can connect Neutron-Antineutron oscillations to Dark Matter problem rather than to Baryogenesis. Infact, if $\psi$ is a metastable fermion of mass $1-1000 \mathrm{TeV}$, an exchange of a virtual exotic vector-like pair can generate $n-\psi$ and $\psi-\bar{n}$ oscillations, with $\tau_{n-\psi} \simeq \tau_{\psi-\bar{n}} \simeq \tau_{n-\bar{n}} / 2 \simeq 10^{8} \mathrm{~s}$. In this case a Neutron-Antineutron transition can be generated as a combination of these two $|\Delta B|=1$ oscillations, without needing of a Majorana mass for Neutron. $\psi$ can be a good candidate of WIMP Dark Matter.

The paper is organized as follows: in section 2, we describe the model for a Majorana neutron also discuss suppression of FCNCs; in section 3, we discuss implications for LHC physics; in section 4, connections with Baryogenesis; in section 5, we discuss a possible string-inspired scenario for the effective model proposed, in section 6 , we present our conclusions.

\section{A model for a neutron Majorana mass}

We introduce a vector-like pair of (complex) color-triplet scalars $\mathcal{X}_{i}, \mathcal{Y}^{i}$ (an their antiparticles) with $i, j$ color indices of $\mathrm{SU}(3)_{c} . \mathcal{X}$ has hypercharge $Y(\mathcal{X})=-2 / 3, \mathcal{Y}$ has hypercharge $Y(\mathcal{Y})=+2 / 3$. Baryon and Lepton numbers are $B(\mathcal{X})=1 / 3, B(\mathcal{Y})=2 / 3$ and $L(\mathcal{X})=L(\mathcal{Y})=0$. We also consider a Majorana sterile particle $\psi(1,1 ; 0)$, with a mass term $\mu \psi \psi+$ h.c. This is a gauge singlet with zero Baryon number, zero Lepton number, zero hypercharge.

\footnotetext{
${ }^{2}$ The see-saw mechanism type I for the neutrino was originally proposed by Minkowski [8], M.GellMann, P.Ramond and R.Slansky [9, 10], by Yanigida [11], R.Mohapatra and G.Senjanovic [12]. Then, other mechanisms called type II [13-17] and type III [14, 15, 18], have been proposed later.

${ }^{3}$ Probably, the most similar mechanism of the one proposed here is in [39-41, 43]. In this case Baryon number is violated by a baryonic 'RH neutron', with a B-violating Majorana mass term.
} 


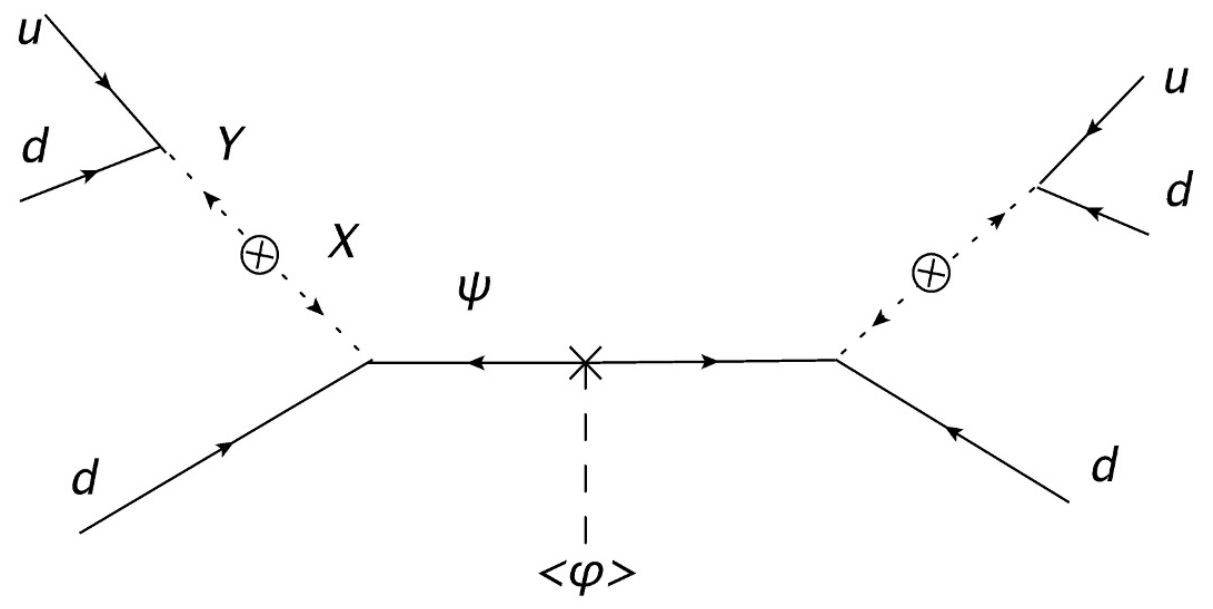

Figure 1. Diagram inducing a Neutron-Antineutron transition. The white blobs indicate the mixing mass term between the vector-like pair of color scalar triplets $\mathcal{X}, \mathcal{Y}$. The central propagator is the Majorana fermion $\psi$.

\begin{tabular}{|l|cc|c|}
\hline Fields & $Y$ & $B$ & $L$ \\
\hline $\mathcal{X}(3,1 ;-2 / 3)$ & $-2 / 3$ & $+1 / 3$ & 0 \\
$\mathcal{Y}(\overline{3}, 1 ;+2 / 3)$ & $+2 / 3$ & $+2 / 3$ & 0 \\
$\psi(1,1 ; 0)$ & 0 & 0 & 0 \\
$q_{L}(3,2 ;+1 / 3)$ & $+1 / 3$ & $+1 / 3$ & 0 \\
$u_{R}(\overline{3}, 1 ;-4 / 3)$ & $-4 / 3$ & $-1 / 3$ & 0 \\
$d_{R}(\overline{3}, 1 ;+2 / 3)$ & $+2 / 3$ & $-1 / 3$ & 0 \\
$l_{L}(1,2 ;-1)$ & -1 & 0 & -1 \\
$e_{R}(1,1 ; 2)$ & +2 & 0 & +2 \\
\hline
\end{tabular}

Table 1. New matter fields introduced with respect to SM. We report their representation with respect to $\mathrm{SM}$ gauge group $\mathrm{SU}(3) \times \mathrm{SU}(2) \times \mathrm{U}(1)_{Y}$, their hypercharges $Y$ and their Baryon and Lepton numbers $B, L$. We also report Standard quarks and leptons for a comparison.

These fields, compatible with gauge invariances, can interact with quark fields as

$$
\mathcal{L}_{Y}=y_{1} \mathcal{X}_{i} \psi d_{R}^{i}+y_{2} \mathcal{Y}^{i} u_{R}^{j} d_{R}^{k} \epsilon_{i j k}+h . c
$$

mass terms for $\mathcal{X}$ and $\mathcal{Y}$,

$$
\mathcal{L}_{\text {mass }}=m_{\mathcal{X}}^{2} \mathcal{X}^{\dagger} \mathcal{X}+m_{\mathcal{Y}}^{2} \mathcal{Y}^{\dagger} \mathcal{Y}+h . c
$$

and $\mathcal{X}-\mathcal{Y}$ has a peculiar mixing mass term

$$
\mathcal{L}_{\mathcal{X}-\mathcal{Y}}=\mathcal{M}_{0}^{2} \mathcal{X}^{i} \mathcal{Y}_{i}+h . c=\frac{1}{2} \mathcal{M}_{0}^{2} \epsilon_{i j k} \mathcal{X}^{i} \mathcal{Y}^{[j k]}+h . c
$$

With these interactions, one can construct a Neutron-Antineutron transitions as shown in figure 1. Note that all interactions terms are B-preserving, exception for mixing term 
$\mathcal{M}_{0}^{2} \epsilon_{i j k} \mathcal{X}^{i} \mathcal{Y}^{[j k]}$, violating baryon number as $\Delta B=1$. Effective operator $(u d d)^{2} / \mathcal{M}^{5}$ has a mass scale $\mathcal{M}=\left(\mathcal{M}_{0}^{4} \mu\right)^{1 / 5}$, times coupling constants $y_{1,2}$, where $\mu$ is mass of fermion $\psi$. Experimental bound on $n-\bar{n}$ implies $\mathcal{M}>300 \mathrm{TeV}$. So, one can consider different choices of parameters $\mathcal{M}_{0}$ and $\mu$ in order to satisfy experimental limits. A trivial choice could be $\mathcal{M}_{0}=\mu=300 \mathrm{TeV}$, automatically saturating the bound. On the other hand, we can also consider for example $\mathcal{M}_{0} \simeq 1-10 \mathrm{TeV}$ and $\mu \simeq 10^{6 \div 10} \mathrm{TeV}$, generating a lot of interesting physics for LHC, as discussed later. Another branch could be $\mu \simeq 1-10^{3} \mathrm{GeV}$ corresponding to $\mathcal{M}_{0} \simeq 7 \times 10^{3 \div 2} \mathrm{TeV}$. In this last case, the fermion $\psi$ is a natural candidate for WIMP dark matter, and Feynman diagram in figure 1 can be seen as a combination of two oscillations $n-\psi$ and $\psi-\bar{n}$ with $\tau_{n \psi} \simeq \tau_{\psi \bar{n}} \simeq 10^{8} \mathrm{~s}: \psi$ is a (meta)stable particle, and not a virtual one in propagator, in this case. Note that actual best limits on $n-\psi$ oscillations are $\tau \geq 414 \mathrm{~s}$, from Ultra Cold Neutron experiments, in condition of suppressed magnetic fields $|\mathcal{B}|<10^{-4}$ Gauss [35-38].

More precisely, in estimation of $\mathcal{M}$, we have to consider not $\mathcal{M}_{0}^{2}$, but the smallest mass eigenvalue of mass matrix of $\mathcal{X}, \mathcal{Y}$. We assume $\mathcal{M}_{0}$ as a real parameter. We can decompose the color complex scalars as $\mathcal{X}=\frac{1}{\sqrt{2}}\left(\mathcal{X}_{1}+i \mathcal{X}_{2}\right)$ and $\mathcal{Y}=\frac{1}{\sqrt{2}}\left(\mathcal{Y}_{1}+i \mathcal{Y}_{2}\right)$, and we can write mass matrix, in basis $\left(\mathcal{X}_{1}, \mathcal{X}_{2}, \mathcal{Y}_{1}, \mathcal{Y}_{2}\right)$ as

$$
M_{\text {eff }}^{2}=\left(\begin{array}{cccc}
m_{\mathcal{X}}^{2} & 0 & \mathcal{M}_{0}^{2} & 0 \\
0 & m_{\mathcal{X}}^{2} & 0 & -\mathcal{M}_{0}^{2} \\
\mathcal{M}_{0}^{2} & 0 & m_{\mathcal{Y}}^{2} & 0 \\
0 & -\mathcal{M}_{0}^{2} & 0 & m_{\mathcal{Y}}^{2}
\end{array}\right)
$$

The eigenvalues are

$$
\lambda_{ \pm}^{2}=\frac{1}{2}\left(m_{\mathcal{X}}^{2}+m_{\mathcal{Y}}^{2} \pm \sqrt{4 \mathcal{M}_{0}^{4}+\left(m_{\mathcal{X}}^{2}-m_{\mathcal{Y}}^{2}\right)^{2}}\right)
$$

(two-two degeneracies, as manifest in (2.4)).

In this model, we are not generating a proton decay process, if the mass of $\psi$ is higher than proton mass. ${ }^{4}$

\subsection{FCNC bounds and the space of the parameters}

FCNC in meson physics are generated in our model. The strongest effects can come from a direct exchange of one $\mathcal{Y}$, shown in figure 2. In particular diagrams (b) in figure 2

\footnotetext{
${ }^{4}$ We assume that other possible interactions of $\mathcal{X}, \mathcal{Y}, \psi$ with leptonic sector are suppressed, in order to avoid other dangerous effective operators. For example, possible extra operators like $\mathcal{Y} q_{\alpha} l^{\alpha}$, leading to a proton decay operator $q q q l / \Lambda^{2}$, can be avoided through opportune discrete symmetry $Z_{N}$, compatible with $\Delta B=1$ operators like $\mathcal{M}_{0} \mathcal{X Y}$. Note that $\mathcal{X}, \mathcal{Y}$ are not leptoquarks, they not have Lepton numbers, in our case. We are assuming that our model is not violating lepton number as $\Delta L=1$; this is simple to realize just with a discrete symmetry $Z_{2}$. This can be also compatible with Majorana masses for neutrini $\Delta L=2$. We also note that $\psi$ is not a Right-handed neutrino, it has a Lepton number equal to zero. For a complete classification of gauge discrete symmetries, protecting the proton by $D=6$ operators, for string constraints on Discrete symmetries, see [51, 52]. Alternatively, in a string-inspired model like [28], R-parity is dynamically broken by Exotic Instantons, generating (2.1)-(2.2)-(2.3), without other dangerous operators. For instance, $q q q \mathrm{l} / \Lambda^{2}$ is automatically avoided! [28].
} 
a)

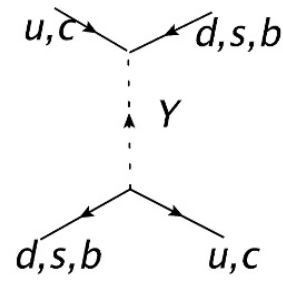

b)

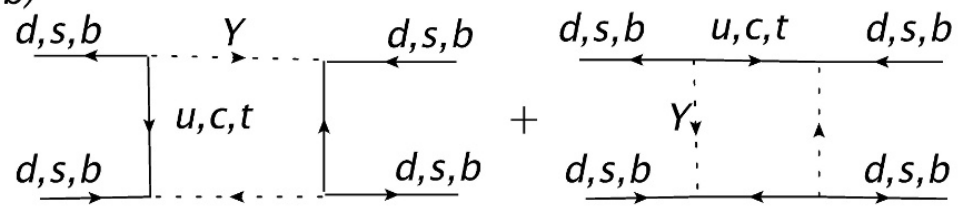

Figure 2. a) FCNCs tree-level diagrams mediated by $\mathcal{Y}$. b) Diagrams of neutral-meson oscillations, mediated by two $\mathcal{Y}$.
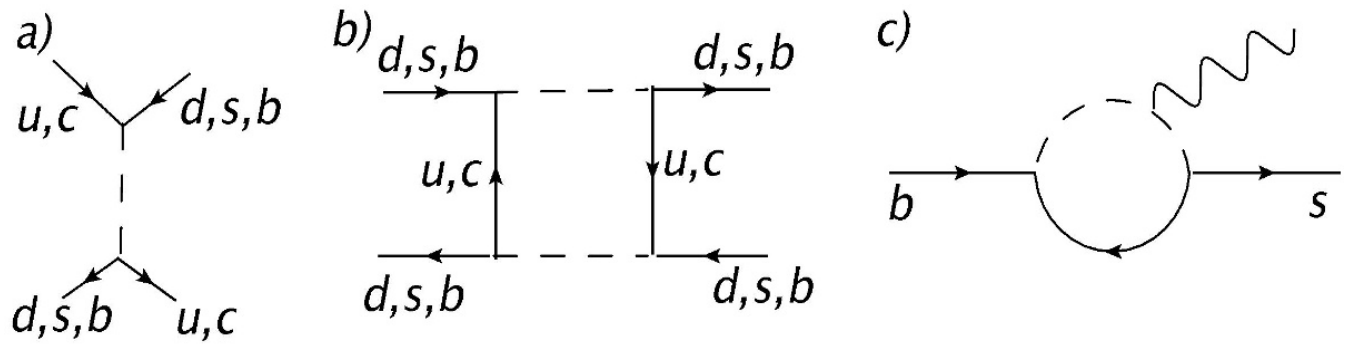

Figure 3. a) Diagram for meson decays into two mesons [28]. This is mediated by two sterile fermions $\psi$ and four $\mathcal{X}-\mathcal{Y}$. b) diagram for neutral meson-antimeson oscillation [28].

a)

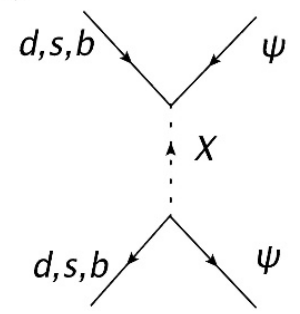

b)

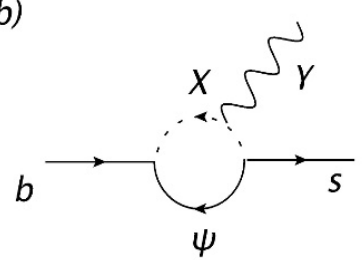

c)

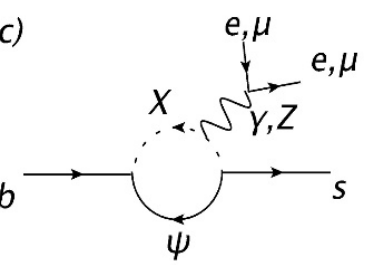

Figure 4. $B^{0}, B_{s}^{0}, \bar{B}^{0}, \bar{B}_{s}^{0} \rightarrow \psi \psi$ are possible if $2 \mu \leq m_{B}$. b) $b \rightarrow s \gamma$ transitions leading to $B \rightarrow K \gamma$ $\phi \gamma$. c) $b \rightarrow s l^{+} l^{-}$transitions leading to $B \rightarrow K l^{+} l^{-}, \phi l^{+} l^{-}$.

contribute to neutral meson-antimeson oscillations such as $K_{0}-\bar{K}_{0}, D_{0}-\bar{D}_{0}, B_{0}-\bar{D}_{0}$ etc. These constrain Y''s mass up to $m_{\mathcal{Y}} \gtrsim 1000 \mathrm{TeV}$. However, these FCNC are not directly constraining $\mathcal{X}$ 's mass. In particular, assuming $m_{\mathcal{Y}}^{2} \simeq 10^{6} m_{\mathcal{X}}^{2}$ and $\mathcal{M}_{0}^{2} \simeq m_{\mathcal{X}}^{2}$, we obtain, from (2.5): $\lambda_{-}^{2} \simeq m_{\mathcal{X}}^{2}$ and $\lambda_{+}^{2} \simeq m_{\mathcal{Y}}^{2}$, with mixing angles $\theta_{13}=\theta_{24} \sim 10^{-6}$. So, mixings between $\mathcal{X}$ and $\mathcal{Y}$ are strongly suppressed in this case, but enough for neutronantineutron transitions: an prefactor of $10^{-12}$ in a $n-\bar{n}$ scale $\left(\mathcal{M}_{0}^{4} \mu\right)^{1 / 5}$. has to be considered. This strongly afflicts estimations of parameters: for $\mathcal{M}_{0}=1-10 \mathrm{TeV}$, it is enough a light $\psi$ of $\mu=1 \div 100 \mathrm{GeV}$ ! As a consequence, the lightest eigenstate of mass matrix (2.5) can elude FCNC's constraints of figure 2 and it can stay also near TeV scale. Other FCNC's contributions, directly involving $\mathcal{X}$, are suppressed, practically avoiding any current observations as shown in figure 3 . In neutral mesons' oscillations $K_{0}-\bar{K}_{0}$, $D_{0}-\bar{D}_{0}, B_{0}-\bar{D}_{0}$ etc. any effects are suppressed as $\mathcal{M}_{0}^{-8} \mu^{-2}$. This strongly motivates a direct research of exotic color scalar triplets (the lightest eigenstate) at LHC. In next 
a)

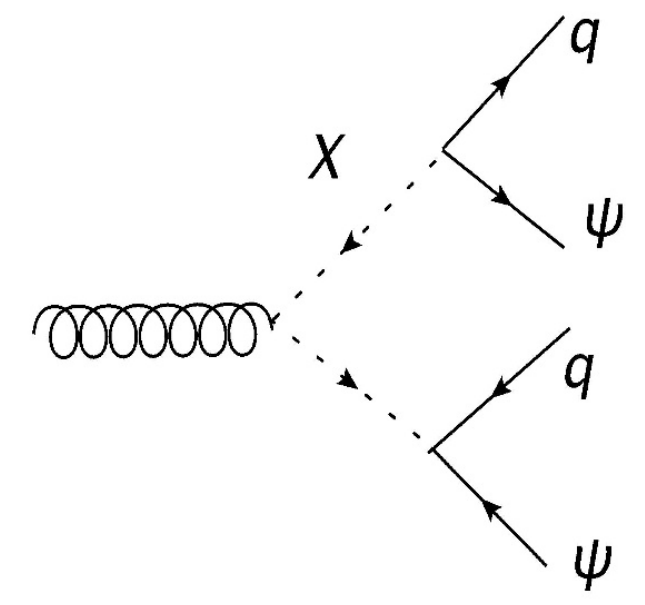

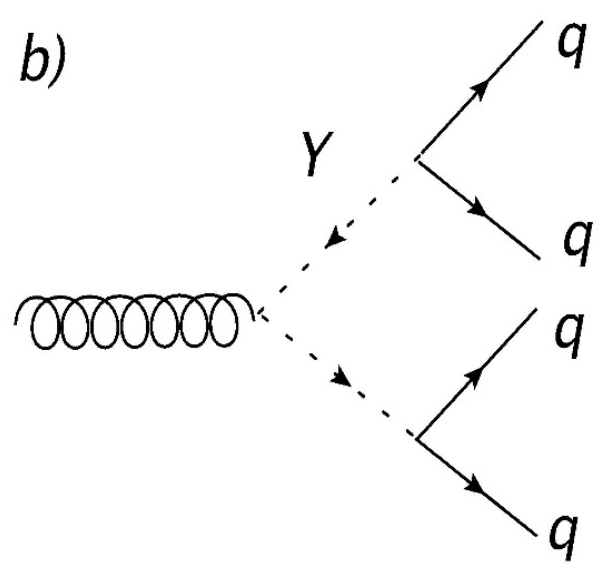

Figure 5. a) Missing energy channel $j j E_{T}$ at LHC; b) Diagram leading to $4 j$ and $t \bar{t} j j$ channels.

section, we will discuss these aspects. We also note that possible decays as $D^{0}, B^{0} \rightarrow \psi \psi$, shown in figure 4 , can be generated if $2 \mu \leq m_{D^{0}, B^{0}}$. Suppose $2 \mu \leq m_{B}$ : in order to satisfy $n-\bar{n}$ limits, $\mathcal{X}$ may have $m_{\mathcal{X}} \gg 1 \mathrm{TeV}$, strongly suppressing decays in figure 4 , or colliders' processes. In the following discussion, we will assume $\mu>m_{B} / 2 \simeq 2.5 \mathrm{GeV}$.

Other effects generated in our model are $b \rightarrow s \gamma$ and $b \rightarrow s l^{+} l^{-}$, shown in (b)-(c) figure 4. Possible deviations in these are predicted in our model, with similar limits of supersymmetric models [50], compatible with limits from the other channels discussed above.

\section{LHC physics}

As discussed in section 2, a direct production of the e.v.l.p is possible: bounds from neutronantineutron physics allow $\mathcal{M}_{0} \sim 1-10 \mathrm{TeV}$. A possible diagram of direct production of the lightest mass eigenstate of $\mathcal{X}-\mathcal{Y}$ is represented in figure 5 -(a). Compatible with FCNCs discussed above, We call two mass eigenstates as $\mathcal{Z}_{ \pm}$, with mass eigenvalues $\lambda_{ \pm}^{2}$. We can reach the lowest eigenstate $\mathcal{Z}_{-}$, with eigenvalue $\lambda_{-} \simeq \mathcal{M}_{0}$, compatible with FCNCs' bounds. For LHC physics, practically $\mathcal{Z}_{-} \simeq \mathcal{X}$. An interesting signature for LHC is $p p \rightarrow j j \not_{T}$. From this channel, we can put limit on $\left(m_{\mathcal{X}}, m_{\psi}\right)$; essentially the same of squarks $\tilde{q} \tilde{q} \rightarrow j j \boldsymbol{E}_{T}$ $[47,48]$. For $m_{\mathcal{X}}>200 \mathrm{GeV} \rightarrow \mu>200 \mathrm{GeV} ; m_{\mathcal{X}}>500 \mathrm{GeV} \rightarrow \mu>400 \mathrm{GeV} ; m_{\mathcal{X}}>$ $1000 \mathrm{GeV} \rightarrow \mu$ is unbounded from below. As a consequence, $\psi$ could be a (meta)stable particle visible at LHC as transverse missing energy and Dark Matter Direct Detection. In this scenario, Neutron-Antineutron physics is directly connected to the Dark Matter question. ${ }^{5}$

We also mention limits from top-jet and di-jets channels, in figure 3-(b), around $1 \mathrm{TeV}$ (top-jet $900 \mathrm{GeV}$, di-jets $1.2 \mathrm{TeV}$ ) [49], but these are not lower than FCNC ones cited above.

\section{Post-sphaleron baryogenesis}

In the proposed mode, one can envisage two simple mechanisms for post-sphaleron baryogenesis: i) $\phi$-decays into six-quarks (antiquarks), ii) $\psi$-decays into three-quarks (antiquarks).

\footnotetext{
${ }^{5}$ If $\psi$ compose all Dark Matter, from WIMP relic abundance $\mu>7 \mathrm{GeV}[44,45]$.
} 


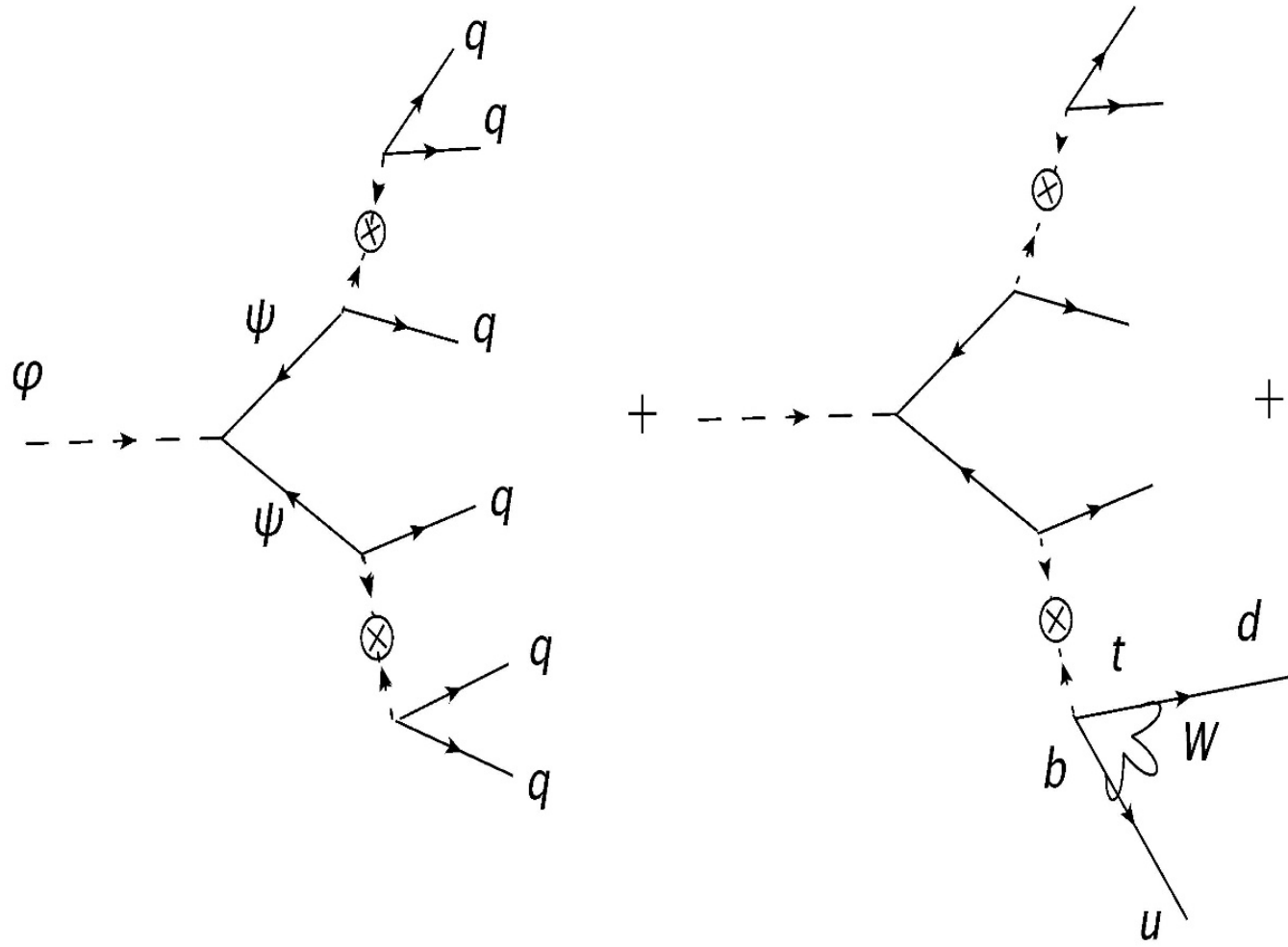

Figure 6. Decay $\phi \rightarrow 6 q$ : the first is a tree level contribution, but also one loops contributions, as the one shown, have to be considered. One-loop contribution in figure is an example of electroweak CKM correction to decay amplitude through an exchange of a $W$ boson, converting top - down and bottom - up. Because of Majorana particle $\psi$, we can revert all arrows in Feynman diagrams, obtaining $\phi \rightarrow 6 \bar{q}$.

We discuss these two in the following.

\subsection{Scalar-decays into six quarks (antiquarks)}

We can reverse diagram in figure 1, considering the mass parameter of $\psi$ as generated by a scalar field $\phi$, acquiring a vev scale $v$, with $\mu=y_{\psi} v$. For the moment, the mass of $\phi$ is a free parameter, $M_{\phi}{ }^{6}$ In figure 6 we show decay diagrams, at tree level and one-loop. We can evaluate the amplitude $\mathcal{M}$, at tree level, as

$$
\mathcal{M}^{\text {tree }} \simeq \frac{y_{\psi}<\phi>\operatorname{Tr}\left[y_{1}^{\dagger} y_{1}\right] \operatorname{Tr}\left[y_{2}^{\dagger} y_{2}\right] \mathcal{V}^{*} \mathcal{V}}{\mathcal{M}_{0}^{4} \mu^{2}}=\frac{\operatorname{Tr}\left[y_{1}^{\dagger} y_{1}\right] \operatorname{Tr}\left[y_{2}^{\dagger} y_{2}\right] \mathcal{V}^{*} \mathcal{V}}{\mathcal{M}_{0}^{4} \mu}
$$

where $\mathcal{V}$ is the diagonalizing matrix of masses (2.4), suppressing the amplitude as $\mathcal{V}^{*} \mathcal{V} \sim$ $10^{-12}$, as cited above. under the assumption $\lambda_{+} \gg \lambda_{-} \simeq \mathcal{M}_{0}$, where $\lambda_{ \pm}$are the mass eigenvalues in (2.5).

\footnotetext{
${ }^{6}$ More precisely we can rewrite $\phi=\left(v+\phi_{r}+\phi_{i}\right) / \sqrt{2}$, and the dynamical scalar decaying is $\phi_{r}$. In the following discussions, for $\phi$-decays we will always mean $\phi_{r}$-decays, and for $M_{\phi}$ we will mean $M_{\phi_{r}}$.
} 
One-loop corrections from the electroweak sector can be evaluated as (assuming all the couplings in $\lambda_{1} \sim \lambda_{2} \sim 10^{-3} \div 1$ )

$$
\mathcal{M}^{1-\text { loop }} \simeq c \mathcal{V}^{*} \mathcal{V} V_{u b}^{*} V_{t d} \Phi_{1-\text { loop }} \overline{\mathcal{O}}_{n \bar{n}}^{2}\left(\frac{\mu m_{t} m_{b}}{m_{W}^{2}}\right)
$$

where $c \simeq\left(10^{-3} \div 1\right)^{4} / 128 \pi^{2}$, and $\overline{\mathcal{O}}_{n \bar{n}}^{2} \equiv<\bar{n}\left|\mathcal{O}^{2}\right| n>\simeq-0.3 \times 10^{-5} \mathrm{GeV}^{6}$ from MIT bag model [30] (confirmed also by recent lattice calculations [31]). $\Phi^{1-l o o p}$ (with dimension mass $\left.\left[\Phi^{1-\text { loop }}\right]=M^{-4}\right)$ is a function depending on the mass of the quarks closing the one-loop in figure 6 (the the top and bottom masses, in dominant contribution). However, there are also other possible contributions, closing 1-loops involving the vector-like pairs, in which $\Phi^{1-l o o p}$ is depending also on vector-like pair. A precise evaluation of such a formula is not necessary for our purposes. It is a good approximation to compare directly (4.1) with the present bounds on neutron-antineutron physics. In principle, we have also to consider running prefactors connecting high energy physics of baryogenesis with low energy neutronantineutron physics. This prefactor is around $10^{-2}$ [32].

At three level, the decay rate of $\phi$ is the square modulus of the amplitude (4.1), times a phase space factor for a $6 q$ (or $6 \bar{q})$ final state:

$$
\Gamma_{\phi}=\Gamma(\phi \rightarrow 6 q)+\Gamma(\phi \rightarrow 6 \bar{q})=\mathcal{I} \mathcal{V}^{*} \mathcal{V} \operatorname{Tr}\left[y_{1}^{\dagger} y_{1}\right]^{2} \operatorname{Tr}\left[y_{2}^{\dagger} y_{2}\right]^{2}\left(\frac{M_{\phi}^{13}}{\mu^{4} \mathcal{M}_{0}^{8}}\right)
$$

with $\mathcal{I} \simeq 7 \times 10^{-18}$ a numerical factor coming from a numerical integration in the phase space times combinatoric factors (practically independent from the ratios of mass parameters, the variations on this integration are of the order of $1 \%$, not important for our purposes).

Considering the case of a Post-sphaleron baryogenesis: the rate (4.3) has to be smaller than the Hubble rate at a temperature near the electroweak phase transition epoch: $\Gamma_{S}<H\left(T_{e w}\right)$. We consider a decay temperature indicatively between $100 \mathrm{GeV} \div 200 \mathrm{MeV}$, between electroweak phase transition and the QCD phase transition $\left(\Lambda_{Q C D} \simeq 200 \mathrm{MeV}\right)$. The decay temperature $\bar{T}$ can be found solving the equation

$$
\Gamma_{S}(\bar{T}) \simeq H(\bar{T}) \simeq 1.66 g_{*}^{1 / 2} \frac{\bar{T}^{2}}{M_{P l}}
$$

where $g_{*}$ is the number of degrees of freedom at $\bar{T}$. From this we can get

$$
\bar{T} \simeq \sqrt{\frac{M_{P l} M_{\phi}^{13}}{(2 \pi)^{9} \mu^{4} \mathcal{M}_{0}^{8}}}
$$

So, a post sphaleron scenario impose limits on the masses' ratios. For example, supposing $\bar{T} \sim 100 \div 200 \mathrm{GeV}$ and $M_{\phi} \simeq 0.5 \mathrm{TeV}$ : we can get bounds on the vector-like pair mixing mass $\mathcal{M}_{0}$ and Majorana fermion mass, well compatible with the ones coming from neutronantineutron physics.

Finally, we can evaluate the primordial baryon asymmetry parameter, directly related to the observed baryon asymmetry:

$$
\epsilon \simeq \frac{n_{\phi}}{n_{\gamma}} \frac{\Gamma(\phi \rightarrow 6 q)-\Gamma(\phi \rightarrow 6 \bar{q})}{\Gamma_{\phi}}
$$


It is necessary to evaluate this including 1-loop CP-violating contributions coming from the electroweak sector, i.e CKM CP violating contributions. The contribution from 1-loop vertices $^{7}$ as the one shown in figure 6 are (considering (4.2))

$$
\epsilon^{V} \simeq \frac{g_{2}^{2}}{32 \pi} \frac{\mathcal{V}^{*} \mathcal{V} y_{2}^{\dagger} V_{t d}^{*} V_{u b} y_{2}}{\operatorname{Tr}\left[y_{2}^{\dagger} y_{2}\right]} \frac{m_{t} m_{b}}{m_{W}^{2}}\left[1+\frac{9 m_{W}^{2}}{M_{\phi}^{2}} \ln \left(1+\frac{\mathrm{M}_{\phi}^{2}}{3 \mathrm{~m}_{\mathrm{W}}^{2}}\right)\right]
$$

With $\epsilon^{V}$ one-loop vertex contribution. So the asymmetry is controlled by $M_{\phi}$. As a consequence, $M_{\phi} \gg 500 \div 1000 \mathrm{GeV}$ suppresses the contribution from the vertex. Cimparing this bound with the other one coming from (4.5), the region of the parameters discussed in section 2 are well compatible. As a consequence, a Post-sphaleron baryogenesis is possible and naturally predicts a neutron-antineutron oscillation of $\tau_{n \bar{n}} \simeq 10^{8}-10^{10} \mathrm{~s}$.

Finally, we also have to consider the dilution of the baryon asymmetry: $\bar{T} \simeq M_{\phi} / 5 \div$ $M_{\phi} / 10$, the decay of $\phi$ generated entropy into the primordial plasma. The dilution can be evaluate as the ratio of entropy density before and after $\phi$-decay:

$$
\mathcal{D}=\frac{s_{\text {initial }}}{s_{\text {final }}} \simeq \frac{0.6 \sqrt{\Gamma_{\phi} M_{P l}}}{g_{*}^{1 / 4} M_{\phi} r_{\phi}}
$$

where $r_{\phi}=n_{\phi} / s$ is at the decays' epoch. This can be estimated as

$$
\mathcal{D} \sim k \frac{\bar{T}}{M_{\phi}} \sim k(10 \% \div 20 \%)
$$

(where $k$ parametrize also extra suppressions from the couplings). From (4.7), we can find $\epsilon \sim 10^{-8 \div 9}$, but this has to be normalized with the dilution factor. We obtain (assuming all couplings near one i.e $k \sim 1), \eta_{B} \sim \mathcal{D} \epsilon \sim 10^{-9 \div 10}$, where $\eta_{B}=\left(n_{b}-n_{\bar{b}}\right) / n_{\gamma}$, as requeired observations $\left(\eta_{B}^{\text {exp }}=(6.04 \pm 0.08) \times 10^{-10}[33]\right)$.

So, we can conclude that this mechanism can generate baryon asymmetry in our Universe, during a Post-Sphaleron epoch, satisfying all Sakharov's conditions i.e i) out of thermal equilibrium; ii) CP-violating processes iii) B-violating processes [46].

\subsection{Majorana fermion decays in three quarks (antiquarks)}

Alternatively, we can consider directly $\psi \rightarrow u_{i} d_{j} d_{k}, \bar{u}_{i} \bar{d}_{j} \bar{d}_{k}$, in which $\mu$ is below electroweak scale. In this scenario, color triplets cannot be detected at LHC. The decay rate can be evaluated as

$$
\Gamma_{\psi \rightarrow q q q, \bar{q} \bar{q} \bar{q}}=c k \mu^{5}\left(\frac{1}{\lambda_{+}^{2}}-\frac{1}{\lambda_{-}^{2}}\right)^{2}
$$

where $\lambda_{ \pm}$are mass eigenvalues in (2.5), and

$$
c=1 / 4096 \pi^{3}, \quad k=\mathcal{V}^{*} \mathcal{V} y_{1}^{\dagger} y_{1} \operatorname{Tr}\left[\mathrm{y}_{2}^{\dagger} \mathrm{y}_{2}\right]
$$

\footnotetext{
${ }^{7}$ One can consider also 1-loop contributions coming involving also $\mathcal{X}, \mathcal{Y}, \psi$ in the propagators. However, one can numerically evaluates these contributions and discover that they are subdominant with respect to the contributions in (4.6). Also Self-energy contributions (or wave-function renormalizations) give not important contributions for our estimations.
} 
a)

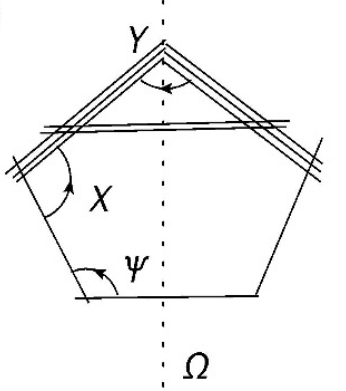

b)

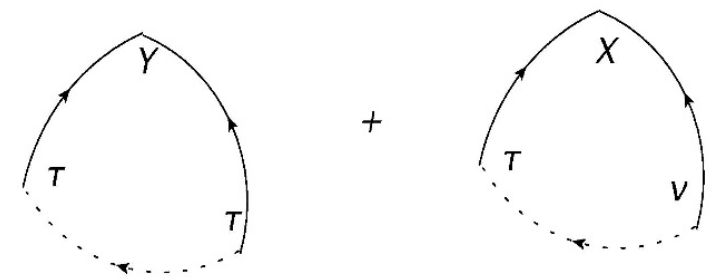

Figure 7. a) (Sub)-system of D-branes stacks generating our toy-model content of fields at low energy limit. b) Mixed-Disk amplitudes generating an Exotic mass term for $\mathcal{X}, \mathcal{Y}$.

(c contains also color factor 6 in numerator). We are assuming $\lambda_{ \pm} \gg \mu$. Under the assumption $\lambda_{+} \gg \lambda_{-} \simeq \mathcal{M}_{0},(4.10)$ is simplified as

$$
\Gamma_{\psi \rightarrow q q q, \bar{q} \bar{q} \bar{q}}=c k \mu^{5} \frac{1}{\mathcal{M}_{0}^{4}}
$$

However, we have also to consider scattering processes. $q+\psi \rightarrow \bar{q} \bar{q}$ : they go-out of equilibrium at the same temperature $\bar{T}$ of $\psi \rightarrow 3 q(\bar{q})$ decays. For $\bar{T}<\mu, \psi$ cannot be produced, for lack of phase space. So, one has also to consider $\psi \psi \rightarrow q \bar{q}$ contributions to baryon asymmetry generation. Extra one-loop electroweak corrections ( $W^{ \pm}$exchanges) lead to dominant contributions as (4.7) cited above. From this, we can estimate $\epsilon \simeq$ $10^{-8} \div 10^{-9}$, for $k \sim 1$ (natural couplings), ulteriorly suppressed by by dilution factor for $10^{-1}$, as discussed in the previous subsection. We conclude that also mechanism seems a viable way to generate the observed Baryon asymmetry.

\section{Beyond the toy-model: string-inspired standard model and exotic in- stantons}

In this section, we would like to discuss a possible explanation of the toy-model, ${ }^{8}$ as a String-Inspired class of model, embedding the Standard Model, generating an exotic mass term for the vector-like pairs We suggest a little different variant with respect to the one suggested in [28]: a IIA (un)-oriented string theory, with stacks of D6 ordinary branes, and Euclidean and D2-branes, wrapping 3-cycles on $C Y_{3}$, and an antisymmetric Mirror Plane $\Omega_{-}$, recovering at low energy limit $\mathrm{U}(3) \times \mathrm{U}(2) \times \mathrm{U}(1) \times U^{\prime}(1)$ or $\mathrm{U}(3) \times \mathrm{Sp}(2) \times \mathrm{U}(1) \times U^{\prime}(1)$, $\mathcal{N}=1$ susy, R-parity preserving. A possible simplified scheme of D-brane stacks (sub)system is shown in figure 7 -(a): $\mathcal{X}, \mathcal{Y}$ are scalar parts of superfields $\mathbf{X}, \mathbf{Y}$, attached between a $\mathrm{U}(3)$ stack and a $\mathrm{U}(1)$ stack, and a $\mathrm{U}(3)$ stack and its mirror twin, with respect the mirror plane $\Omega$, respectively. On the other hand, $\psi$ is the fermionic part of a superfield $\Psi$ living between two U(1) stacks. Finally, also $\phi$ can be constructed, similarly to $\psi$. We can introduce an Exotic E2-brane intersecting with ordinary ones. In this way, we generate interactions between Grassmann moduli (or modulini), living between $E 2-D 6$

\footnotetext{
${ }^{8}$ We mention that, recently, a toy model for a supersymmetric non-local QFT was discussed in [57].
} 


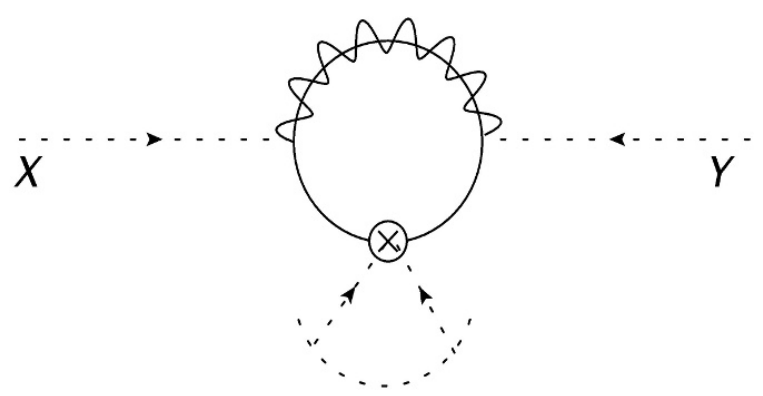

Figure 8. Exotic mass for $\mathcal{X}, \mathcal{Y}$, generated by one-loop corrections, containing one gaugino, and a $\psi_{\mathcal{X}, \mathcal{Y}}$ mixing induced by Exotic Instantons (white blob with dashed lines).

intersections, and ordinary superfields. Let us discuss the consistency of the hypercharges in a construction like the one suggested in figure 7. For intersecting D-brane model considered in figure $7, \mathrm{U}(1)_{Y}$ is defined as a linear of $\mathrm{U}(1)$ stacks:

$$
\mathrm{U}(1)_{Y}=c_{1} \mathrm{U}(1)_{1}+c_{1}^{\prime} U^{\prime}(1)+c_{3} \mathrm{U}(1)_{3}
$$

where $\mathrm{U}(1)_{2} \subset \mathrm{U}(2), \mathrm{U}(1)_{2} \subset \mathrm{U}(2)$. So the hypercharge is a combination of four abelian charges. From (5.1), a consistent assignation of hypercharges, $Y(\mathcal{X})=-Y(\mathcal{Y})=-2 / 3$, $Y(\Psi)=0$, and the ones of SM particles, can be found. In particular, we find $c_{3}=1 / 3$, $c_{1}=c_{1}^{\prime}=-1$.

As in [28], a non-perturbative mass term between $\mathbf{X}, \mathbf{Y}$ can be generated by two mixed-disk amplitudes, shown in figure 7-(b). In fact, from these,

$$
\mathcal{L}_{E 2-D 6-D 6^{\prime \prime}} \sim \nu \tau^{i} \mathbf{X}_{i}+\mathbf{Y}_{i j} \tau^{i} \tau^{j}
$$

where $i, j$ are the color indices of the $\mathrm{U}(3)$-stack. A new superpotential term, not allowed at perturbative level, is obtained, integrating out modulini:

$$
\mathcal{W}_{E 2}=M_{S} e^{-S_{E 2}} \int d^{3} \tau d \omega e^{\nu \tau^{i} \mathbf{X}_{i}+\mathbf{Y}_{i j} \tau^{i} \tau^{j}}=M_{S} e^{-S_{E 2}} \epsilon_{i j k} \mathbf{X}^{i} \mathbf{Y}^{j k}
$$

where $M_{S}$ is the String scale and $e^{-S_{E 2}}$ is the parameterize by geometric moduli of the 3-cycles wrapped by the Euclidean D2-brane in the Calabi-Yau $C Y_{3}$. As shown figure 8, an exotic mass term can be generated, in a supersymmetric model, as a loop of susy partners $\psi_{\mathcal{X}}, \psi_{\mathcal{Y}}$ and a gaugino (gluino, zino or photino), with $\mathcal{M}_{0}^{2} \sim m_{\tilde{g}} M_{S} e^{-S_{E 2}}, m_{\tilde{g}}$ gaugino mass.

We would like to note that all contributions on irreducible gauge anomalies, cancel each other, in this D-brane construction. In fact, $\mathcal{X}, \mathcal{Y}$ do not introduce extra anomalous contributions with respect to SM fields content. For instance, $\mathrm{SU}(3)^{3}$ anomalies give equal and opposite contributions because of $\operatorname{Tr}[\mathcal{X}]=1$ and $\operatorname{Tr}[\mathcal{Y}]=N_{c}-4=-1$. On the other hand, anomalous extra $\mathrm{U}(1)$ are introduced with respect to SM gauge group: new $Z^{\prime}$ are introduced as in any string-inspired model, with masses generated by a Stückelberg mechanism $[58,59]$. Anomalies that could appear as a serious problem in gauge models, 
are cancelled by Generalized Chern-Simons (GCS) terms as a generalized Green-Schwarz mechanism $[60,61]^{9}$

Finally, we would like to remark that, an exotic mass term (5.3) cannot be introduced by-hand, at perturbative level, because of R-parity, i.e R-parity is dynamically broken, without the generation of other dangerous R-parity violating operators, as explained in $[28,29,66]$.

\section{Conclusions}

In this paper, we have discussed a simple alternative model generating a Majorana mass for the neutron, connecting Majorana's proposal to deep issues regarding Baryogenesis and Dark Matter. In particular, we have introduced just one exotic vector-like pair of colortriplet scalars, a sterile Majorana fermion $\psi$, and a scalar giving mass to $\psi$. An exotic vector-like pair is characterized by an extra peculiar mass term, violating baryon number by $\Delta B=1$. In particular, we got limits on exotic mixing mass parameter from LHC physics. We have seen how Baryogenesis can be realized, also during the post-sphaleron epoch, and we predict a neutron-antineutron transition with a time interesting for the next generation of experiments: $\tau_{n \bar{n}} \sim 300 \mathrm{yr}$. We have also considered, an alternative scenario, in which the sterile fermion is a metastable WIMP-like particle. In this case, a neutronantineutron transition can be generated by two $\Delta B=1$ oscillations, $n-\psi$ and $\psi-\bar{n}$. Finally, we have also shown a possible completion and explanation of such a toy-model, in which the exotic mass term is generated by non-perturbative exotic stringy istantons.

We conclude that this model, postulating an exotic vector-like pair of color-triplet scalars, deserves attention for its peculiarity and simplicity, especially considering its possible connections with fundamental issues and its implications in B-violations phenomenology such as neutron-antineutron physics and LHC.

\section{Acknowledgments}

A.A would like to thanks Galileo Galilei Institute for Theoretical Physics for the hospitality, where this paper was prepared. I would like to thank Massimo Bianchi, Zurab Berezhiani and Luca Di Luzio for interesting conversations. I also would like to thank the anonymous referee for his important comments and suggestions. The work of A.A. was supported in part by the MIUR research grant "Theoretical Astroparticle Physics" PRIN 2012CPPYP7.

Open Access. This article is distributed under the terms of the Creative Commons Attribution License (CC-BY 4.0), which permits any use, distribution and reproduction in any medium, provided the original author(s) and source are credited.

\footnotetext{
${ }^{9}$ The Stückelberg mechanism has a lot of different intriguing applications. Let us mention that a Lorentz Violating Massive gravity can be realized through a Stückelberg mechanism [62-64]. Recently, geodetic instabilities of Stückelberg Lorentz Violating Massive gravity were discussed in [65].
} 


\section{References}

[1] E. Majorana, Theory of the symmetry of electrons and positrons, Nuovo Cim. 14 (1937) 171 [INSPIRE].

[2] V.A. Kuzmin, CP-noninvairiance and baryon asymmetry of the universe, JETP Lett. 12 (1970) 228.

[3] S.L. Glashow, Overview, in Proceedings of Neutrino 79, International conference on neutrinos, weak interactions and cosmology, 1 (1979), pg. 518 [INSPIRE].

[4] R.N. Mohapatra and R.E. Marshak, Local B-L symmetry of electroweak interactions, Majorana neutrinos and neutron oscillations, Phys. Rev. Lett. 44 (1980) 1316 [INSPIRE].

[5] M. Baldo-Ceolin et al., A new experimental limit on neutron-anti-neutron oscillations, $Z$. Phys. C 63 (1994) 409 [INSPIRE].

[6] Particle Data Group collaboration, J. Beringer et al., Review of particle physics (RPP), Phys. Rev. D 86 (2012) 010001 [INSPIRE].

[7] I. Phillips et al., Neutron-antineutron oscillations: theoretical status and experimental prospects, submitted to Phys. Rept. (2014) [arXiv:1410.1100] [INSPIRE].

[8] P. Minkowski, $\mu \rightarrow$ er at a rate of one out of $10^{9}$ muon decays?, Phys. Lett. B 67 (1977) 421 [INSPIRE].

[9] P. Ramond, The family group in grand unified theories, hep-ph/9809459 [INSPIRE].

[10] M. Gell-Mann, P. Ramond and R. Slansky, Complex spinors and unified theories, Conf. Proc. C 790927 (1979) 315 [arXiv:1306.4669] [InSPIRE].

[11] T. Yanagida, Horizontal symmetry and masses of neutrinos, Prog. Theor. Phys. 64 (1980) 1103 [INSPIRE].

[12] R.N. Mohapatra and G. Senjanović, Neutrino mass and spontaneous parity violation, Phys. Rev. Lett. 44 (1980) 912 [inSPIRE].

[13] M. Magg and C. Wetterich, Neutrino mass problem and gauge hierarchy, Phys. Lett. B 94 (1980) 61 [INSPIRE].

[14] G. Lazarides, Q. Shafi and C. Wetterich, Proton lifetime and fermion masses in an $\mathrm{SO}(10)$ model, Nucl. Phys. B 181 (1981) 287 [InSPIRE].

[15] R.N. Mohapatra and G. Senjanović, Neutrino masses and mixings in gauge models with spontaneous parity violation, Phys. Rev. D 23 (1981) 165 [INSPIRE].

[16] R.N. Mohapatra and P. Pal, Massive neutrinos in physics and astrophysics, World Scientific, Singapore (1991), pg. 127.

[17] E. Ma and U. Sarkar, Neutrino masses and leptogenesis with heavy Higgs triplets, Phys. Rev. Lett. 80 (1998) 5716 [hep-ph/9802445] [INSPIRE].

[18] S.M. Barr, A different seesaw formula for neutrino masses, Phys. Rev. Lett. 92 (2004) 101601 [hep-ph/0309152] [INSPIRE].

[19] C.H. Albright and S.M. Barr, Leptogenesis in the type-III seesaw mechanism, Phys. Rev. D 69 (2004) 073010 [hep-ph/0312224] [INSPIRE].

[20] K.S. Babu, P.S. Bhupal Dev, E.C. F.S. Fortes and R.N. Mohapatra, Expectations for neutron-antineutron oscillation time from TeV scale baryogenesis, AIP Conf. Proc. 1534 (2012) 211 [INSPIRE]. 
[21] K.S. Babu and R.N. Mohapatra, Coupling unification, GUT-scale baryogenesis and neutron-antineutron oscillation in SO(10), Phys. Lett. B 715 (2012) 328 [arXiv:1206.5701] [INSPIRE].

[22] K.S. Babu, P.S. Bhupal Dev, E.C. F.S. Fortes and R.N. Mohapatra, Post-sphaleron baryogenesis and an upper limit on the neutron-antineutron oscillation time, Phys. Rev. D 87 (2013) 115019 [arXiv:1303.6918] [INSPIRE].

[23] S. Patra and P. Pritimita, Post-sphaleron baryogenesis and $n-\bar{n}$ oscillation in non-SUSY $\mathrm{SO}(10)$ GUT with gauge coupling unification and proton decay, Eur. Phys. J. C $\mathbf{7 4}$ (2014) 3078 [arXiv: 1405.6836] [INSPIRE].

[24] J.M. Arnold, B. Fornal and M.B. Wise, Simplified models with baryon number violation but no proton decay, Phys. Rev. D 87 (2013) 075004 [arXiv:1212.4556] [inSPIRE].

[25] G. Dvali, Three-form gauging of axion symmetries and gravity, hep-th/0507215 [INSPIRE].

[26] C. Cheung and K. Ishiwata, Baryogenesis with higher dimension operators, Phys. Rev. D 88 (2013) 017901 [arXiv: 1304.0468] [INSPIRE].

[27] P. Fileviez Perez, New paradigm for baryon and lepton number violation, arXiv:1501.01886 [INSPIRE].

[28] A. Addazi and M. Bianchi, Neutron Majorana mass from exotic instantons, JHEP 12 (2014) 089 [arXiv: 1407.2897] [INSPIRE].

[29] A. Addazi and M. Bianchi, Un-oriented quiver theories for Majorana neutrons, arXiv: 1502.01531 [INSPIRE].

[30] S. Rao and R. Shrock, $n \leftrightarrow \bar{n}$ transition operators and their matrix elements in the MIT bag model, Phys. Lett. B 116 (1982) 238 [INSPIRE].

[31] M.I. Buchoff, C. Schroeder and J. Wasem, Neutron-antineutron oscillations on the lattice, PoS(LATTICE 2012) 128 [arXiv: 1207.3832] [INSPIRE].

[32] P.T. Winslow and J.N. Ng, Neutron-antineutron oscillations in a warped extra dimension, Phys. Rev. D 81 (2010) 106010 [arXiv:1003.1424] [inSPIRE].

[33] Planck collaboration, P.A.R. Ade et al., Planck 2013 results. XVI. Cosmological parameters, Astron. Astrophys. 571 (2014) A16 [arXiv:1303.5076] [INSPIRE].

[34] R. Sekhar Chivukula, E.H. Simmons and N. Vignaroli, Distinguishing dijet resonances at the LHC, Phys. Rev. D 91 (2015) 055019 [arXiv:1412.3094] [INSPIRE].

[35] A.P. Serebrov et al., Experimental search for neutron: mirror neutron oscillations using storage of ultracold neutrons, Phys. Lett. B 663 (2008) 181 [arXiv:0706.3600] [INSPIRE].

[36] I. Altarev et al., Neutron to mirror-neutron oscillations in the presence of mirror magnetic fields, Phys. Rev. D 80 (2009) 032003 [arXiv: 0905.4208] [InSPIRE].

[37] K. Bodek et al., Additional results from the first dedicated search for neutronmirror neutron oscillations, Nucl. Instrum. Meth. A 611 (2009) 141 [INSPIRE].

[38] Particle Data Group collaboration, K. Nakamura et al., Review of particle physics, J. Phys. G 37 (2010) 075021 [inSPIRE].

[39] Z. Berezhiani and L. Bento, Neutron-mirror neutron oscillations: how fast might they be?, Phys. Rev. Lett. 96 (2006) 081801 [hep-ph/0507031] [INSPIRE]. 
[40] Z. Berezhiani and L. Bento, Fast neutron: mirror neutron oscillation and ultra high energy cosmic rays, Phys. Lett. B 635 (2006) 253 [hep-ph/0602227] [INSPIRE].

[41] Z. Berezhiani, More about neutron-mirror neutron oscillation, Eur. Phys. J. C 64 (2009) 421 [arXiv: 0804.2088] [INSPIRE].

[42] A. Addazi, Neutron-Antineutron oscillations as a test of a new interaction, to appear in Proceedings of IFAE 2014, Italy (2014).

[43] A. Addazi, Z. Berezhiani and Y. Kamyshkov, Neutron-antineutron oscillations as a test of a new fundamental interaction, in preparation.

[44] A. Bottino, F. Donato, N. Fornengo and S. Scopel, Indirect signals from light neutralinos in supersymmetric models without gaugino mass unification, Phys. Rev. D 70 (2004) 015005 [hep-ph/0401186] [INSPIRE].

[45] N. Fornengo, S. Scopel and A. Bottino, Discussing direct search of dark matter particles in the minimal supersymmetric extension of the standard model with light neutralinos, Phys. Rev. D 83 (2011) 015001 [arXiv: 1011.4743] [INSPIRE].

[46] A.D. Sakharov, Violation of CP invariance, $c$ asymmetry and baryon asymmetry of the universe, Pisma Zh. Eksp. Teor. Fiz. 5 (1967) 32 [JETP Lett. 5 (1967) 24] [Sov. Phys. Usp. 34 (1991) 392] [Usp. Fiz. Nauk 161 (1991) 61] [INSPIRE].

[47] ATLAS collaboration, Search for squarks and gluinos with the ATLAS detector in final states with jets and missing transverse momentum using $\sqrt{s}=8$ TeV proton-proton collision data, JHEP 09 (2014) 176 [arXiv:1405.7875] [INSPIRE].

[48] CMS collaboration, Search for new physics in the multijet and missing transverse momentum final state in proton-proton collisions at $\sqrt{s}=8 \mathrm{TeV}$, JHEP 06 (2014) 055 [arXiv: 1402.4770] [INSPIRE].

[49] ATLAS collaboration, Exotics public results webpage, https://twiki.cern.ch/twiki/bin/view/AtlasPublic/ExoticsPublicResults.

[50] K. Kowalska, Phenomenology of SUSY with general flavour violation, JHEP 09 (2014) 139 [arXiv: 1406.0710] [INSPIRE].

[51] M. Berasaluce-Gonzalez, L.E. Ibáñez, P. Soler and A.M. Uranga, Discrete gauge symmetries in D-brane models, JHEP 12 (2011) 113 [arXiv:1106.4169] [INSPIRE].

[52] P. Anastasopoulos, M. Cvetič, R. Richter and P.K.S. Vaudrevange, String constraints on discrete symmetries in MSSM type II quivers, JHEP 03 (2013) 011 [arXiv:1211.1017] [INSPIRE].

[53] R. Blumenhagen, M. Cvetič and T. Weigand, Spacetime instanton corrections in $4 D$ string vacua: the seesaw mechanism for D-brane models, Nucl. Phys. B 771 (2007) 113 [hep-th/0609191] [INSPIRE].

[54] L.E. Ibáñez and A.M. Uranga, Neutrino Majorana masses from string theory instanton effects, JHEP 03 (2007) 052 [hep-th/0609213] [INSPIRE].

[55] L.E. Ibáñez, A.N. Schellekens and A.M. Uranga, Instanton induced neutrino Majorana masses in CFT orientifolds with MSSM-like spectra, JHEP 06 (2007) 011 [arXiv: 0704.1079] [INSPIRE].

[56] S. Antusch, L.E. Ibáñez and T. Macri, Neutrino masses and mixings from string theory instantons, JHEP 09 (2007) 087 [arXiv:0706.2132] [INSPIRE]. 
[57] A. Addazi and G. Esposito, Nonlocal quantum field theory without acausality and nonunitarity at quantum level: is SUSY the key?, arXiv:1502.01471 [INSPIRE].

[58] B. Körs and P. Nath, A Stueckelberg extension of the standard model, Phys. Lett. B 586 (2004) 366 [hep-ph/0402047] [INSPIRE].

[59] B. Körs and P. Nath, Aspects of the Stueckelberg extension, JHEP 07 (2005) 069 [hep-ph/0503208] [INSPIRE].

[60] J. De Rydt, J. Rosseel, T.T. Schmidt, A. Van Proeyen and M. Zagermann, Symplectic structure of $N=1$ supergravity with anomalies and Chern-Simons terms, Class. Quant. Grav. 24 (2007) 5201 [arXiv:0705.4216] [inSPIRE].

[61] D. Feldman, Z. Liu and P. Nath, The Stueckelberg Z' extension with kinetic mixing and milli-charged dark matter from the hidden sector, Phys. Rev. D 75 (2007) 115001 [hep-ph/0702123] [INSPIRE].

[62] N. Arkani-Hamed, H. Georgi and M.D. Schwartz, Effective field theory for massive gravitons and gravity in theory space, Annals Phys. 305 (2003) 96 [hep-th/0210184] [INSPIRE].

[63] V.A. Rubakov, Lorentz-violating graviton masses: getting around ghosts, low strong coupling scale and VDVZ discontinuity, hep-th/0407104 [INSPIRE].

[64] S.L. Dubovsky, Phases of massive gravity, JHEP 10 (2004) 076 [hep-th/0409124] [INSPIRE].

[65] A. Addazi and S. Capozziello, External stability for spherically symmetric solutions in Lorentz breaking massive gravity, arXiv:1407.4840 [INSPIRE].

[66] A. Addazi and M. Bianchi, Neutron Majorana mass from exotic instantons in a Pati-Salam model, arXiv:1502.08041 [INSPIRE]. 